\title{
Optimal antithrombotic treatment of patients with atrial fibrillation undergoing percutaneous coronary intervention: triple therapy is too much!
}

\author{
M. S. Jacobs ${ }^{1,2} \cdot$ R. G. Tieleman ${ }^{3,4}$ \\ Published online: 8 May 2018 \\ (c) The Author(s) 2018
}

\begin{abstract}
Patients with atrial fibrillation who undergo a coronary intervention are eligible for both anticoagulation and (dual) antiplatelet therapy ((D)APT). An optimal balance has to be found to reduce the thromboembolic risk (i.e. stroke, systemic embolism and myocardial infarction) and to minimise the increased risk of bleeding with concomitant use of an anticoagulant and (D)APT. Owing to a lack of evidence, the guideline recommendations are predominantly based on expert opinion. Current evidence indicates that the combination of a non-vitamin K oral anticoagulant (NOAC) and clopidogrel is safer than vitamin-K oral antagonists plus DAPT, which increases the risk of bleeding, without clear advantages in regard to efficacy. Concerning whether $(N)$ OACs should be combined with single APT rather than DAPT, the findings of the WOEST, PIONEER AF-PCI and RE-DUAL PCI trials seem to favour a combination with clopidogrel only, thus omitting aspirin. Choosing the optimal treatment strategies for individual patients on NOACs and (D)APT will remain a challenge for clinicians, though triple therapy seems to be the less favourable option owing to the increased risk of bleeding.
\end{abstract}

Keywords Anticoagulation - Antiplatelet therapy $\cdot$ Atrial fibrillation $\cdot$ Acute coronary syndrome $\cdot$ Coronary intervention

\section{Introduction}

Almost $30 \%$ of patients with atrial fibrillation (AF) have co-existing coronary artery disease (CAD) $[1,2]$. Anticoagulants are prescribed to AF patients to reduce the risk of stroke or systemic embolism. However, there is still a risk of the patient developing acute coronary syndrome (ACS), requiring percutaneous coronary intervention (PCI). Following PCI, antiplatelet drugs such as aspirin and P2Y12 inhibitors (clopidogrel, prasugrel and ticagrelor) play a predominant role in the prevention of in-stent thrombosis and

R. G. Tieleman

r.tieleman@mzh.nl

1 Department of Clinical Pharmacy and Toxicology, Martini Hospital, Groningen, The Netherlands

2 Groningen Research Institute of Pharmacy, Unit of PharmacoTherapy, Epidemiology \& Economics (PTEE), University of Groningen, Groningen, The Netherlands

3 Department of Cardiology, Martini Hospital, Groningen, The Netherlands

4 Department of Cardiology, University Medical Center Groningen, University of Groningen, Groningen, The Netherlands cardiovascular events [3]. Antiplatelet therapy (APT) alone is inferior to oral anticoagulation for reducing the risk of thromboembolic events in AF patients [4]. According to the present European Society of Cardiology (ESC) guidelines, AF patients with co-existing ACS and those who undergo a coronary intervention are eligible for both anticoagulation and (dual) antiplatelet therapy ((D)APT) [5, 6]. However, a combination of warfarin with (D)APT carries a more than threefold higher risk for non-fatal and fatal bleeding compared with warfarin monotherapy [7]. An optimal balance has to be found to reduce the thromboembolic risk (i.e. stroke, systemic embolism and myocardial infarction) and to minimise the increased risk of bleeding resulting from concomitant use of an anticoagulant and (D)APT. Owing to the lack of evidence, the 2016 ESC guideline on the treatment of ACS in AF patients and the 2017 ESC focused update on DAPT in CAD are predominantly based on expert opinion $[3,6,8]$. This review summarises and discusses the most recent key developments and future studies with regard to combining anticoagulation (non-vitamin $\mathrm{K}$ oral anticoagulants (NOACs) or vitamin K antagonists (VKA)) with APT in AF patients undergoing PCI. 
KEY MESSAGE

- Single antiplatelet therapy with clopidogrel only, thus omitting aspirin, in combination with oral anticoagulation is safer than dual antiplatelet therapy in atrial fibrillation (AF) patients who undergo a coronary intervention. Therefore, triple antithrombotic therapy seems to be too much.

- The lowest dose of the non-vitamin $\mathrm{K}$ oral antagonist suitable for stroke prevention in $\mathrm{AF}$ patients should be used in combination with antiplatelet therapy. Dose adjustments, or even withholding anticoagulation, should not be decided solely on the basis of the bleeding risk.

- All ongoing trials on NOACs in combination with antiplatelet therapy are primarily focused on the safety endpoint in terms of major bleeding or clinically relevant non-major bleeding. The trials are underpowered to draw firm conclusions about the efficacy for stroke prevention in AF.

\section{Guideline recommendations and current practice}

The 2014 AF guideline for the management of AF patients of the American College of Cardiology/American Heart Association Task Force on Practice Guidelines and the Heart Rhythm Society (AHA/ACC/HRS) recommends warfarin rather than NOACs as the first-choice therapy in AF patients with ACS. This guideline mentions considering dual therapy consisting of an OAC plus clopidogrel $75 \mathrm{mg}$ once daily (q.d.) as an alternative to initial triple therapy [5].

The 2015 ESC non-ST segment elevation myocardial infarction (NSTEMI) guideline recommends that the use of prasugrel or ticagrelor as a part of triple therapy should be avoided in the absence of safety and efficacy data (class C recommendation) [3]. Because it is known that the addition of (D)APT to oral coagulation increases the bleeding risk, limited data suggest that clopidogrel is probably the safest of the available P2Y12 inhibitors because of its lowest bleeding risk [9]. If the OAC is a VKA, the ESC NSTEMI guideline recommends a target international normalisation ratio (INR) of 2.0-2.5 with the exception of patients with a mechanical prosthetic valve in the mitral position [3].

The 2016 ESC guideline for AF recommends a short period of triple therapy (OAC + aspirin + clopidogrel) followed by a period of dual therapy (OAC + APT, preferably up to 12 months after the event) in AF patients with co-existing ACS and those who undergo PCI [6]. The duration of the dual and triple therapy depends on the bleeding risk as calculated by the HAS-BLED score, the reason for the PCI (ACS or stable CAD) and the type of stent (bare-metal versus drug-eluting). Modifiable bleeding risk factors should be corrected to minimise the risk of bleeding. This guideline also mentions dual therapy with an OAC and clopidogrel as an emerging alternative to triple therapy based on the results of the WOEST study (What is the Optimal antiplatElet and anticoagulant therapy in patients with oral anticoagulation and coronary StenTing) [10] When a NOAC is preferred for anticoagulation, the lowest effective dose for stroke pre- vention should be used (apixaban $5 \mathrm{mg}$ twice daily (b.i.d.), dabigatran $110 \mathrm{mg}$ b.i.d., edoxaban $60 \mathrm{mg}$ q.d., rivaroxaban $20 \mathrm{mg}$ q.d.) or the appropriate reduced dose if indicated by dose-reduction criteria according to the drug labelling [2].

Fairly recently, a new ESC focused update was published on DAPT in CAD [8]. The guideline expresses a preference for clopidogrel as a part of triple antithrombotic therapy; prasugrel and ticagrelor should be avoided in combination with oral anticoagulation because of worrisome bleeding rates in registries (level $\mathrm{C}$ recommendation). In patients treated with a NOAC, the lowest effective dose for stroke prevention is also recommended in this guideline. Interestingly, the most innovative recommendation in this guideline is the use of rivaroxaban $15 \mathrm{mg}$ q.d. as an alternative to rivaroxaban $20 \mathrm{mg}$ q.d. when combined with aspirin and/or clopidogrel. The new guideline seems to base this level $\mathrm{B}$ recommendation on the results of the PIONEER AF-PCI (Open-Label, Randomized, Controlled, Multicenter Study Exploring Two Treatment Strategies of Rivaroxaban and a Dose-Adjusted Oral Vitamin K Antagonist Treatment Strategy in Subjects with Atrial Fibrillation who Undergo Percutaneous Coronary Intervention) study. It is noteworthy that this same guideline states that the PIONEER AF-PCI study is largely underpowered for the assessment of meaningful differences in the incidence of relevant ischaemic events, such as stent thrombosis or stroke rates. Dual therapy with clopidogrel $75 \mathrm{mg}$ and oral anticoagulation is still recommended for consideration as an alternative to 1 month of triple therapy for patients in whom the bleeding risk outweighs the ischaemic risk [8].

The European Heart Rhythm Association conducted a survey in 2014 to provide an insight into current practice in Europe regarding the management of AF patients presenting with ACS. This survey showed that, in $91.1 \%$ of cases, combining warfarin with aspirin and clopidogrel was preferable in AF patients after PCI [11]. Most of the responding centres treated patients with triple therapy $(\mathrm{OAC}+$ aspirin + clopidogrel) for 3 months followed by 12 months of dual therapy (OAC + aspirin), and the ma- 
jority used warfarin $[11,12]$. Of the NOACs, rivaroxaban $15 \mathrm{mg}$ o.d. and apixaban $2.5 \mathrm{mg}$ b.i.d. were most frequently used.

\section{Triple vs dual antithrombotic therapy}

\section{Post hoc analyses}

In the post hoc analysis of the RE-LY (Randomized Evaluation of Long-Term Anticoagulation Therapy) trial, dabigatran $110 \mathrm{mg}$ b.i.d. plus (D)APT was not inferior to warfarin plus (D)APT in reducing stroke and systemic embolism and was associated with fewer major bleeds. There was an additive effect on major bleeding risk with the number of antiplatelet drugs used regardless of the OAC dose used, but (D)APT did not reduce the risk of thromboembolism or stroke [13]. A subanalysis of the ENGAGE AF-TIMI 48 (Effective Anticoagulation with Factor Xa Next Generation in Atrial Fibrillation-Thrombolysis in Myocardial Infarction 48) trial also looked at the combination of a NOAC plus APT [14]. Concomitant use of edoxaban $60 \mathrm{mg}$ q.d. or $30 \mathrm{mg}$ q.d. plus APT was compared with warfarin plus APT in AF patients. The study showed that combining APT with edoxaban or warfarin increased the bleeding risk. There was less major bleeding in patients that used edoxaban than in those using warfarin. Moreover, APT did not influence the efficacy of edoxaban compared to warfarin in preventing stroke or systemic embolic events. A predefined analysis of the ARISTOTLE (Apixaban for Reduction in Stroke and Other Thromboembolic Events in Atrial Fibrillation) trial assessed the effect of concomitant aspirin use on the efficacy and safety of apixaban compared with warfarin in AF patients [15]. Apixaban use resulted in similar reductions in stroke and systemic embolism among aspirin users and non-users, compared with warfarin. Apixaban had a consistent effect among aspirin users and non-users on stroke or systemic embolism, ischaemic stroke, myocardial infarction, and death both in the overall population and among the subgroups of patients with and without a history of arterial vascular disease. There were statistically significant reductions in major bleeding for aspirin combined with apixaban compared to warfarin plus aspirin. The effect of apixaban in causing less major bleeding, haemorrhagic stroke, major or clinically relevant non-major bleeding and any bleeding was comparable in subgroups with and without a history of arterial vascular disease. Only $0.7 \%$ of the total population of the ARISTOTLE trial underwent triple therapy (aspirin + P2Y12 receptor antagonist + study drug) for at least 7 days. There was no subanalysis for AF patients with ACS or patients who underwent PCI.

\section{Results of (N)OACs combined with (D) APT in AF patients}

The WOEST study compared the safety of triple therapy (VKA (target INR 2.0) with clopidogrel and aspirin) with that of dual therapy (VKA with clopidogrel) in patients that used an OAC and underwent PCI. This study showed that omitting aspirin significantly reduced bleeding complications (hazard ratio (HR) $0.36,95 \%$ confidence interval (CI) $0.26-0.50, p<0.0001$ ) and was associated with a lower risk of the combined secondary endpoint consisting of death, myocardial infarction, stroke, target-vessel revascularisation and stent thrombosis (HR $0.60,95 \%$ CI $0.38-0.94$ ) [10]. It should be noted that the WOEST study was not powered to detect differences in the occurrence of thrombotic events when aspirin was omitted.

The PIONEER AF-PCI trial [16] compared two different rivaroxaban treatment strategies with a VKA treatment strategy in patients with AF undergoing PCI. The drug regimens were based on the results of the ATLAS ACS 2-TIMI 51 study (Anti-Xa Therapy to Lower Cardiovascular Events in Addition to Standard Therapy in Subjects with Acute Coronary Syndrome-Thrombolysis in Myocardial Infarction 51) and the results of the WOEST study. The PIONEER AF-PCI included the more potent P2Y12 inhibitors ticagrelor and prasugrel as a treatment option, with the choice of the P2Y12 inhibitor at the discretion of the physician. However, over $93 \%$ of the patients were treated with clopidogrel, and therefore no statements per type of P2Y12 inhibitor can be made. The PIONEER trial results demonstrated that use of rivaroxaban $15 \mathrm{mg}$ q.d. plus a P2Y12 inhibitor for 12 months and rivaroxaban $2.5 \mathrm{mg}$ b.i.d. plus DAPT for 1,6 or 12 months was associated with lower rates of clinically significant bleeding compared to VKA plus DAPT for 1,6 or 12 months $(16.8 \%$ for rivaroxaban $15 \mathrm{mg}$ q.d. plus $\mathrm{P} 2 \mathrm{Y} 12$ inhibitor, $18.0 \%$ for rivaroxaban $2.5 \mathrm{mg}$ b.i.d. plus DAPT, $26.7 \%$ for VKA plus DAPT; $\mathrm{HR}=0.59,95 \% \mathrm{CI}=0.47-0.76$ for rivaroxaban $15 \mathrm{mg}$ q.d. plus P2Y12 inhibitor vs VKA+DAPT and $\mathrm{HR}=0.63,95 \% \mathrm{CI}=0.50-0.80$ for rivaroxaban $2.5 \mathrm{mg}$ b.i.d. plus DAPT vs VKA+DAPT). The rates of the prespecified endpoint death from cardiovascular causes, myocardial infarction or stroke were similar in the three groups [16]. Notably, although the stroke rate was not different in the three groups, the study was underpowered to draw any conclusion about the effect on stroke prevention, and the $2.5 \mathrm{mg}$ dose of rivaroxaban was not tested for stroke prevention in AF. The post hoc analysis of the PIONEER AF-PCI trial demonstrated that AF patients who underwent PCI and were treated with rivaroxaban $15 \mathrm{mg}$ q.d. plus a P2Y12 inhibitor for 12 months or rivaroxaban $2.5 \mathrm{mg}$ b.i.d. plus DAPT for 1, 6 or 12 months had a lower risk of all-cause mortality or recurrent hospitalisation for adverse 
events compared to those with VKA plus DAPT for 1,6 or 12 months [17].

The RE-DUAL PCI trial (Randomized Evaluation of Dual Therapy with Dabigatran vs Triple Therapy with Warfarin in Patients with Atrial Fibrillation That Undergo a Percutaneous Coronary Intervention with Stenting) compared dual therapy consisting of dabigatran $(110 \mathrm{mg}$ and $150 \mathrm{mg}$ b.i.d.) and clopidogrel or ticagrelor with triple therapy consisting of warfarin, aspirin and clopidogrel or ticagrelor in patients with non-valvular atrial fibrillation that underwent a PCI with stenting [18]. In this trial, no distinction is made between the specific P2Y12 inhibitors and the duration of (D)APT treatment, and only $12 \%$ received ticagrelor. The average age of the patients was 70.8 years, and ACS was the most frequent PCI indication (50.5\%). The incidence of major or clinically relevant non-major bleeding events during follow-up was significantly lower for dabigatran plus P2Y12 than for triple therapy (HR $0.52,95 \%$ CI $0.42-0.63$ for dabigatran $110 \mathrm{mg}$ and HR $0.72,95 \%$ CI $0.58-0.88$ for dabigatran $150 \mathrm{mg}$ ). The two dual-therapy groups were not inferior with respect to the incidence of the composite efficacy endpoint (myocardial infarction, stroke, systemic embolism, death or unplanned revascularisation) as compared with the triple-therapy group. In contrast to the PIONEER AF-PCI trial, the NOAC doses in the RE-DUAL PCI trial (dabigatran 150 and $110 \mathrm{mg}$ b.i.d.) have both been proved to be effective for stroke prevention in AF. Unfortunately, as only a small number of patients were enrolled per dabigatran dose group, the trial was not powered to examine the efficacy according to dose. Also, the trial was not powered to allow for comparisons of individual components of the efficacy endpoint.

Overall, the results from the WOEST, PIONEER AF-PCI and the RE-DUAL PCI trials suggest that in AF patients who recently underwent PCI, dual therapy with a combination of (N)OAC and clopidogrel is safer than triple therapy with OAC plus aspirin and clopidogrel. The post hoc analysis of the RE-LY study demonstrated that dabigatran $110 \mathrm{mg}$ b.i.d. plus DAPT (aspirin and clopidogrel) is safer than VKA plus DAPT in terms of bleeding. Whether this is also true for dabigatran plus clopidogrel versus VKA plus clopidogrel cannot be concluded from the study results published thus far. It should be noted that the RE-DUAL and PIONEER AF-PCI studies as well as the post-analyses of previous studies had a target INR of 2.0-3.0, which is higher than the guideline recommendation of 2.0-2.5. The time in therapeutic range (TTR) is a very import aspect to take into account, since a low TTR could influence the clinical outcomes.

\section{Ongoing trials}

A summary of the multiple ongoing trials evaluating the role of NOACs and (D)APT in AF patients with co-existing ACS is presented in Tab. 1. The AUGUSTUS trial (an Openlabel, $2 \times 2$ Factorial, Randomized Controlled, Clinical Trial to Evaluate the Safety of Apixaban vs Vitamin K Antagonist and Aspirin vs Aspirin Placebo in Patients with Atrial Fibrillation and Acute Coronary Syndrome or Percutaneous Coronary Intervention; NTC02415400) compares the safety of apixaban $5 \mathrm{mg}$ (or $2.5 \mathrm{mg}$ ) b.i.d. with VKA both with and without aspirin in patients with AF and ACS or PCI. All patients in this study will also be taking a P2Y12 inhibitor. Because of its $2 \times 2$ factorial design, this study will provide insight into the safety of apixaban versus a VKA when both are combined with single APT. Moreover, it will address the safety and efficacy of triple versus dual therapy in a randomised design: OAC (VKA/NOAC) with DAPT versus OAC (VKA/NOAC) with single APT. The safety and efficacy of edoxaban in AF patients who undergo PCI with stenting will be investigated in the ENTRUST-AFPCI trial (Edoxaban Treatment Versus Vitamin K Antagonist in Patients with Atrial Fibrillation Undergoing Percutaneous Coronary Intervention; NTC02866175). This study will compare the safety and efficacy of $60 \mathrm{mg}$ or $30 \mathrm{mg}$ edoxaban plus clopidogrel or another P2Y12 inhibitor with a VKA plus clopidogrel and 1-12 months of aspirin (in the presence of a documented clinical need: prasugrel or ticagrelor) [19]. The ongoing trial investigating rivaroxaban in combination with APT in AF patients with ACS is the RT-AF trial (Rivaroxaban in Patients with Atrial Fibrillation and Coronary Artery Disease Undergoing Percutaneous Coronary Intervention; NTC02334254). The RT-AF trial evaluates the safety of rivaroxaban $2.5 \mathrm{mg}$ b.i.d. plus ticagrelor versus triple therapy with warfarin plus clopidogrel and aspirin [20]. Safety is assessed based on a composite primary endpoint of major bleeding and clinically relevant non-major bleeding. The last ongoing trial which will provide information about the management of NOACs in AF patients with ACS is the WOEST 2 registry (NTC0263520). This study is a prospective, international registry on concomitant use of OACs and P2Y12 inhibitors in patients with AF or heart valve prosthesis undergoing coronary revascularisation. This study will provide insight into the efficacy and safety of all possible combinations of NOACs and antiplatelet inhibitors.

Most of the treatment arms of the ongoing trials focus on one single NOAC combined with a single antiplatelet drug. Based on the design of the trials, superiority or even non-inferiority of combining a single antiplatelet drug with a NOAC compared to DAPT plus a NOAC cannot be demonstrated, with the exception of the AUGUSTUS trial, where apixaban plus clopidogrel plus aspirin will be 
Table 1 Overview of ongoing trials on concomitant use of non-vitamin $\mathrm{K}$ oral anticoagulants and antiplatelet drugs in atrial fibrillation patients with an acute coronary syndrome

\begin{tabular}{|c|c|c|c|c|}
\hline Trial & AUGUSTUS & ENTRUST-AF-PCI & RT-AF & WOEST 2 registry \\
\hline Study design & $\begin{array}{l}\text { Open-label, } 2 \times 2 \text { factorial, } \\
\text { randomized controlled clinical } \\
\text { trial }\end{array}$ & $\begin{array}{l}\text { Randomized, open label, } \\
\text { safety study }\end{array}$ & $\begin{array}{l}\text { Open-label, randomized } \\
\text { controlled clinical trial }\end{array}$ & $\begin{array}{l}\text { Prospective, international, } \\
\text { multi-center, non-interven- } \\
\text { tional, cohort study }\end{array}$ \\
\hline Objective & $\begin{array}{l}\text { To assess the safety of the } \\
\text { different treatment arms in AF } \\
\text { patients undergoing PCI }\end{array}$ & $\begin{array}{l}\text { To assess the safety of the } \\
\text { different treatment arms } \\
\text { in AF patients undergoing } \\
\text { PCI }\end{array}$ & $\begin{array}{l}\text { To assess the safety of the } \\
\text { different treatment arms } \\
\text { in AF patients undergoing } \\
\text { PCI }\end{array}$ & $\begin{array}{l}\text { To assess the different man- } \\
\text { agement patterns and safety } \\
\text { and efficacy outcomes of } \\
\text { combined use of OAC and } \\
\text { a P2Y12 inhibitor in patients } \\
\text { with AF and/or a heart valve } \\
\text { prosthesis undergoing PCI }\end{array}$ \\
\hline Pt number & 4,600 & 1,500 & 420 & 2,200 \\
\hline Follow-up & 6 months & 24 months & 12 months & 24 months \\
\hline $\begin{array}{l}\text { Estimated } \\
\text { completion }\end{array}$ & December 2018 & February 2019 & January 2016 & December 2019 \\
\hline \multirow[t]{4}{*}{ Arms } & $\begin{array}{l}\text { Apixaban } 5 \mathrm{mg} \text { (or } 2.5 \mathrm{mg} \\
\text { according to dose reduction } \\
\text { criteria) b.i.d. + P2Y } 12 \text { in- } \\
\text { hibitor + ASA } 81 \mathrm{mg} \text { q.d. }\end{array}$ & $\begin{array}{l}\text { Edoxaban } 60 \mathrm{mg} \text { q.d. (or } \\
\text { edoxaban } 30 \mathrm{mg} \text { q.d ac- } \\
\text { cording to dose reduc- } \\
\text { tion criteria) + clopidogrel } \\
75 \text { mg q.d. }\end{array}$ & \multirow{2}{*}{$\begin{array}{l}\text { Rivaroxaban } 2.5 \mathrm{mg} / 5 \mathrm{mg} \\
\text { b.i.d. + ticagrelor } 90 \mathrm{mg} \\
\text { b.i.d. Antiplatelet therapy } \\
\text { is mandatory at least } \\
1 \text { month after BMS } \\
\text { implantation, and } \\
6 \text { months after DES } \\
\text { implantation }\end{array}$} & \multirow[t]{4}{*}{$\begin{array}{l}\text { All combinations of chronic } \\
\text { OAC and a P2Y12 inhibitor } \\
\text { with or without ASA }\end{array}$} \\
\hline & $\begin{array}{l}\text { Apixaban } 5 \mathrm{mg} \text { (or } \\
2.5 \mathrm{mg} \text { ) b.i.d. + P } 2 \text { Y } 12 \text { in- } \\
\text { hibitor + Placebo }\end{array}$ & $\begin{array}{l}\text { VKA + clopidogrel } 75 \mathrm{mg} \\
\text { q.d. + ASA } 100 \mathrm{mg} \\
30 \text { days to } 12 \text { months }\end{array}$ & & \\
\hline & $\begin{array}{l}\text { VKA + P2Y12 inhibitor + ASA } \\
81 \text { mg q.d. }\end{array}$ & \multirow{2}{*}{$\begin{array}{l}\text { In case of a documented } \\
\text { clinical need: prasugrel [ } 5 \\
\text { or } 10 \mathrm{mg} \text { q.d.] or ticagrelor } \\
\text { [90 mg b.i.d.] }\end{array}$} & \multirow{2}{*}{$\begin{array}{l}\text { Rivaroxaban } 2.5 \mathrm{mg} / 5 \mathrm{mg} \\
\text { b.i.d. + ticagrelor } 90 \mathrm{mg} \\
\text { b.i.d. Antiplatelet therapy } \\
\text { is mandatory at least } 1 \\
\text { month after BMS } \\
\text { implantation, and } \\
6 \text { months after DES } \\
\text { implantation }\end{array}$} & \\
\hline & $\begin{array}{l}\mathrm{VKA}+\mathrm{P} 2 \mathrm{Y} 12 \text { in- } \\
\text { hibitor + placebo }\end{array}$ & & & \\
\hline $\begin{array}{l}\text { Primary } \\
\text { outcome }\end{array}$ & $\begin{array}{l}\text { Time to ISTH major or } \\
\text { CRNM bleeding during the } \\
\text { treatment period }\end{array}$ & $\begin{array}{l}\text { Number of major or } \\
\text { CRNM ISTH-bleeding }\end{array}$ & $\begin{array}{l}\text { Number of major or } \\
\text { CRNM bleeding }\end{array}$ & $\begin{array}{l}\text { Composite of thrombotic } \\
\text { events (MI, stroke, TIA, SE) } \\
\text { and cardiovascular death and } \\
\text { Bleeding events }\end{array}$ \\
\hline
\end{tabular}

$A F$ atrial fibrillation, $A S A$ aspirin, b.i.d. twice daily, $B M S$ bare metal stent, $C R N M$ Clinically Relevant Non-Major, $D E S$ drug-eluting stent, INR international normalized ratio, ISTH International Society on Thrombosis and Hemostasis, $M I$ myocardial infarction, NVAF, OAC oral anticoagulant, q.d. once daily, $P C I$ percutaneous coronary intervention, $S E$ systemic embolism; TIA transient ischemic attack, VKA vitamin K antagonist

compared with apixaban plus clopidogrel plus placebo. Of the ongoing trials, RT-AF is the only one that uses a lower target INR (1.8-2.5). None of these trials are performing a head-to-head comparison of different NOACs and therefore provide no insight into the difference in efficacy and safety of these agents.

\section{Discussion}

All of the ongoing trials are primarily focused on the safety endpoint in terms of major bleeding or clinically relevant non-major bleeding. All of these trials are underpowered to draw firm conclusions about the efficacy for stroke prevention in AF. This is of importance, since some of the trials (i.e. PIONEER AF-PCI and RT-AF) use a suboptimal
NOAC dose for stroke prevention in AF. The short followup period of all the studies limits their ability to assess the effect on stroke and venous thromboembolic events, especially in the long term. Furthermore, these studies are also underpowered for the clinical endpoints death, myocardial infarction and in-stent thrombosis in patients with ACS. Most of the studies do not include patients who have had a stroke or bleeding, although these events do not contraindicate NOAC use. When determining the optimal treatment for an individual patient, the clinician should always take into account bleeding risk factors. Modifiable bleeding risk factors are, for example: adding a proton pump inhibitor with DAPT to reduce the risk of gastrointestinal bleeding, hypertension $(<160 \mathrm{~mm} \mathrm{Hg}$ target), alcohol use and the use of predisposing co-medication such as non-steroidal antiinflammatory drugs. 
Despite the efforts of researchers to synthesise more evidence from the ongoing trials, there will still be challenges for clinicians to choose the optimal treatment strategies for individual patients on NOACs and (D)APT with AF undergoing PCI. The ESC guidelines and the AHA/ACC/HRS recommendation to use the lowest effective NOAC dose in combination with (D)APT in patients with AF and co-existing ACS or PCI were based on limited evidence concerning the optimal combination of NOACs and APT (level of evidence $C$; expert opinion) $[3,5,8]$. In this respect, the term "lowest effective dose" could be confusing and the term "lowest suitable dose for AF" would be more appropriate. Current evidence indicates that the combination of NOAC and clopidogrel is safer than VKA plus DAPT, which increases the risk of bleeding, without clear advantages in efficacy.

As to whether (N)OAC should be combined with single APT rather than dual APT, evidence from the WOEST, PIONEER AF-PCI and RE-DUAL PCI trials seems to favour a combination with clopidogrel only, thus omitting aspirin [21]. In this respect, a very interesting trial would involve a treatment arm with only an OAC in an adequate dose for stroke prevention in AF without APT. Unfortunately, this has not been incorporated into any of the studies. A direct comparison of clopidogrel versus aspirin as APT after PCI has not been studied. Evidence from a randomised controlled trial in patients with atherosclerotic vascular disease (manifested as either ischaemic stroke, myocardial infarction or symptomatic peripheral artery disease) showed that clopidogrel was more effective than aspirin in reducing the combined risk of ischaemic stroke/myocardial infarction and vascular death [22]. A nationwide registry study confirmed that there was a marginally significant reduction of MI and coronary death with OAC plus clopidogrel compared to triple therapy in AF patients after myocardial infarction or PCI. This reduction was not significant for OAC plus aspirin [23]. Also, OAC plus aspirin was associated with a significantly increased risk of death from all causes. The choice of clopidogrel over aspirin in ongoing trials seems to be based mainly on the WOEST trial results. None of the studies thus far has made a direct comparison of a VKA plus single APT versus a NOAC plus single APT. The upcoming results of the AUGUSTUS trial will therefore be interesting owing to its $2 \times 2$ factorial design (see Tab. 1). The reduced-dose NOACs should only be given if the dose reduction is indicated according to the patients' characteristics as listed in the drug labelling, irrespective of the APT used. A reduced-dose NOAC in "healthy" patients, with the exception of dabigatran $110 \mathrm{mg}$ q.d., has not been shown to be effective for stroke prevention in AF. A common mistake is to give apixaban $2.5 \mathrm{mg}$ combined with APT instead of the full dose. However, this would only be appropriate in the presence of dose-reduction criteria (creatinine clearance $10-30 \mathrm{ml} / \mathrm{min}$ or two out of three following: serum creatinine $\geq 133 \mu \mathrm{mol} / \mathrm{l}$, body weight $\leq 60 \mathrm{~kg}$ and/or age $\geq 80$ years). The recommendation of the 2017 ESC guideline on DAPT to consider rivaroxaban $15 \mathrm{mg}$ q.d. instead of $20 \mathrm{mg}$ when combined with aspirin and/or clopidogrel should therefore be followed with caution because this dose has not been proved to be effective for stroke prevention in patients with AF. Consequently, rivaroxaban $2.5 \mathrm{mg}$ is not an option for stroke prevention in ACS patients with AF

The majority of the above-mentioned NOAC trials are still recruiting at present, and the results will become available in the coming years. For now, triple therapy has proved to be too much in terms of safety outcomes. However, it can be expected that guidelines will continue to change in the near future, and what seems wise today may become obsolete tomorrow.

Conflict of interest R.G. Tieleman reports a significant research grant from Boehringer Ingelheim and modest honoraria from Boehringer Ingelheim, Bayer, Bristol-Myers-Squibb, and Pfizer. M.S. Jacobs declares no competing interests.

Open Access This article is distributed under the terms of the Creative Commons Attribution 4.0 International License (http:// creativecommons.org/licenses/by/4.0/), which permits unrestricted use, distribution, and reproduction in any medium, provided you give appropriate credit to the original author(s) and the source, provide a link to the Creative Commons license, and indicate if changes were made.

\section{References}

1. Lip GY, Laroche C, Popescu MI, et al. Improved outcomes with European Society of Cardiology guideline-adherent antithrombotic treatment in high-risk patients with atrial fibrillation: a report from the EORP-AF General Pilot Registry. Europace. 2015;17:1777-86.

2. Kirchhof P, Ammentorp B, Darius H, et al. Management of atrial fibrillation in seven European countries after the publication of the 2010 ESC Guidelines on atrial fibrillation: primary results of the PREvention oF thromboemolic events-European Registry in Atrial Fibrillation (PREFER in AF). Europace. 2014;16:6-14.

3. Roffi M, Patrono C, Collet JP, et al. 2015 ESC Guidelines for the management of acute coronary syndromes in patients presenting without persistent ST-segment elevation: Task Force for the Management of Acute Coronary Syndromes in Patients Presenting without Persistent ST-Segment Elevation of the European Society of Cardiology (ESC). Eur Heart J. 2016;37:267-315.

4. ACTIVE Writing Group of the ACTIVE Investigators, Conolly S, Pogue J, Hart R, et al. Clopidogrel plus aspirin versus oral anticoagulation for atrial fibrillation in the Atrial fibrillation Clopidogrel Trial with Irbesartan for prevention of Vascular Events (ACTIVE W): a randomised controlled trial. Lancet. 2006;367:1903-12.

5. ACC/AHA Task Force Members, January CT, Wann LS, Alpert JS, et al. 2014 AHA/ACC/HRS guideline for the management of patients with atrial fibrillation: a report of the American College of Cardiology/American Heart Association Task Force on practice guidelines and the Heart Rhythm Society. Circulation. 2014;130:e199-267. 
6. Kirchhof P, Benussi S, Kotecha D, et al. 2016 ESC guidelines for the management of atrial fibrillation developed in collaboration with EACTS. Eur Heart J. 2016;37:2893-962.

7. Hansen ML, Sorensen R, Clausen MT, et al. Risk of bleeding with single, dual, or triple therapy with warfarin, aspirin, and clopidogrel in patients with atrial fibrillation. Arch Intern Med. 2010;170:1433-41.

8. Valgimigli M, Bueno H, Byrne RA, et al. 2017 ESC focused update on dual antiplatelet therapy in coronary artery disease developed in collaboration with EACTS: the Task Force for dual antiplatelet therapy in coronary artery disease of the European Society of Cardiology (ESC) and of the European Association for Cardio-Thoracic Surgery (EACTS). Eur Heart J. 2017; https://doi.org/10.1093/ eurheartj/ehx419.

9. Bavishi C, Panwar S, Messerli FH, Bangalore S. Meta-analysis of comparison of the newer oral P2Y12 inhibitors (prasugrel or ticagrelor) to clopidogrel in patients with non-ST-elevation acute coronary syndrome. Am J Cardiol. 2015;116:809-17.

10. Dewilde WJ, Oirbans T, Verheugt FW, et al. WOEST study investigators. Use of clopidogrel with or without aspirin in patients taking oral anticoagulant therapy and undergoing percutaneous coronary intervention: an open-label, randomised, controlled trial. Lancet. 2013;381:1107-15.

11. Potpara TS, Lip GY, Dagres N, et al. Conducted by the Scientific Initiatives Committee, European Heart Rhythm Association. Management of acute coronary syndrome in patients with non-valvular atrial fibrillation: results of the European Heart Rhythm Association Survey. Europace. 2014;16:293-8.

12. Scientific Initiative Committee, European Heart Rhythm Association, Larsen TB, Potpara T, Dagres N, et al. Preference for oral anticoagulation therapy for patients with atrial fibrillation in Europe in different clinical situations: results of the European Heart Rhythm Association Survey. Europace. 2015;17:819-24.

13. Dans AL, Connolly SJ, Wallentin L, et al. Concomitant use of antiplatelet therapy with dabigatran or warfarin in the Randomized Evaluation of Long-Term Anticoagulation Therapy (RE-LY) trial. Circulation. 2013;127:634-40.

14. Xu H, Ruff CT, Giugliano RP, et al. Concomitant use of single antiplatelet therapy with edoxaban or warfarin in patients with atrial fibrillation: analysis from the ENGAGE AF-TIMI48 trial. J Am Heart Assoc. 2016;5 https://doi.org/10.1161/JAHA.115.002587.
15. Alexander JH, Lopes RD, Thomas L, et al. Apixaban vs. warfarin with concomitant aspirin in patients with atrial fibrillation: insights from the ARISTOTLE trial. Eur Heart J. 2014;35:224-32.

16. Gibson CM, Mehran R, Bode C, et al. An open-label, randomized, controlled, multicenter study exploring two treatment strategies of rivaroxaban and a dose-adjusted oral vitamin $\mathrm{K}$ antagonist treatment strategy in subjects with atrial fibrillation who undergo percutaneous coronary intervention (PIONEER AF-PCI). Am Heart J. 2015;169:472-478.e5.

17. Gibson CM, Pinto DS, Chi G, et al. Recurrent hospitalization among patients with atrial fibrillation undergoing intracoronary stenting treated with 2 treatment strategies of rivaroxaban or a doseadjusted oral vitamin $\mathrm{k}$ antagonist treatment strategy. Circulation. 2017;135:323-33.

18. Cannon CP, Bhatt DL, Oldgren J, et al. RE-DUAL PCI Steering Committee and Investigators. Dual antithrombotic therapy with dabigatran after PCI in atrial fibrillation. N Engl J Med. 2017; https://doi.org/10.1056/NEJMoa1708454.

19. Vranckx P, Lewalter T, Valgimigli M, et al. Evaluation of the safety and efficacy of an edoxaban-based antithrombotic regimen in patients with atrial fibrillation following successful percutaneous coronary intervention (PCI) with stent placement: rationale and design of the ENTRUST-AF PCI trial. Am Heart J. 2018;196:105-12.

20. Gao F, Shen H, Wang ZJ, Yang SW, Liu XL, Zhou YJ. Rationale and design of the RT-AF study: combination of rivaroxaban and ticagrelor in patients with atrial fibrillation and coronary artery disease undergoing percutaneous coronary intervention. Contemp Clin Trials. 2015;43:129-32.

21. Pareek M, Bhatt DL, Ten Berg JM, Kristensen SD, Grove EL. Antithrombotic strategies for preventing long-term major adverse cardiovascular events in patients with non-valvular atrial fibrillation who undergo percutaneous coronary intervention. Expert Opin Pharmacother. 2017; 18:875-83.

22. CAPRIE Steering Committee. A randomised, blinded, trial of clopidogrel versus aspirin in patients at risk of ischaemic events (CAPRIE). CAPRIE Steering Committee. Lancet. 1996;348:1329-39.

23. Lamberts M, Gislason GH, Olesen JB, et al. Oral anticoagulation and antiplatelets in atrial fibrillation patients after myocardial infarction and coronary intervention. J Am Coll Cardiol. 2013;62:981-9. 